\title{
HIGH-RESOLUTION SURFACE TEXTURE MEASUREMENTS USING A COLLABORATIVE PICK-AND-PLACE ROBOT
}

\author{
M. Riedl ${ }^{1 *}$, R. Danzl ${ }^{1}$, T. Lankmair ${ }^{1}$, F. Millonig ${ }^{1}$ \\ ${ }^{1}$ Alicona Imaging $\mathrm{GmbH}$, Raaba, Austria \\ *Corresponding author; e-mail: matthias.riedl@bruker.com
}

\begin{abstract}
The ongoing trend of robotics, automation, quality control and its combination are leading to new possibilities in the field of metrology. The improvements, especially in the handling and accuracy of collaborative robotics, are enabling new applications for automated high-resolution optical 3D measurements. This is the basis for the implementation of a pick-and-place solution for automatic surface texture measurements by replacing a high precision $x-y$ stage with a collaborative robot.

In this paper two different Focus-Variation setups are compared to evaluate the uncertainty using a certified standard and a real sample.
\end{abstract}

\section{Keywords:}

Inline, collaborative, automation, 3D measurement, optical metrology, 3D dataset, integration, robot, Focus Variation, Pick-and-place, surface texture

\section{INTRODUCTION}

The increasing automation of industrial machining with simultaneous miniaturisation and reduction of production tolerances places the highest demands on quality assurance. Surface and shape parameters must be recorded with ever higher resolution and accuracy. It goes without saying that the measurements must be robust, repeatable, traceable and at the same time fast in an industrial environment. An ever-increasing focus on process automation is also a strong driver for innovation.

These efforts have received additional impetus since collaborative robotics has become more prevalent in industry and the initial safety concerns of companies and employees have been minimised through experience and by standards. Here the human interaction of the user and the robot are possible without the risk of injuries and this leads to new possibilities regarding automated measurement systems [ISO 15066: 2016] [Lankmair et al. 2018] [Zaborowski et al. 2019].

For many applications in combination with collaborative robotics, conventional tactile methods are reaching their limits. Optical and thus non-contact techniques are increasingly being used as an alternative because the sensitivity regarding the imprecise positioning of the robot systems is considerably lower [ISO 9283: 1998]. Frequently used systems are based on white light interferometry, structured light, confocal microscopy, or Focus-Variation [Leach 2011] [Leach 2014] [Weckenmann 2012]. Here, Focus-Variation is particularly suitable because its lower susceptibility to vibrations and also a constant resolution over the larger measuring range which might be necessary due to the positioning inaccuracy of the robot system [Danzl et al. 2011].

For the testing of the capabilities of the measurement design a UR5e manufactured by Universal Robots with a collaborative gripper was used for a Pick-and-place solution with a Focus-Variation sensor head. The measurement workflow was fully automized to perform surface texture measurements without any user influence. The samples were taken out of the sample storage, placed under the sensor head and the measurement was performed. To compare the results a similar Focus-Variation sensor head was used but the positioning was done by a high precision xy stage using encoders. The used samples for both measurement setups were a standard calibrated by the NPL and a titanium turbine blade sample.

\section{COLLABORATIVE PICK-AND-PLACE WITH A FOCUS-VARIATION SENSOR}

\subsection{D Measurement using Focus-Variation}

The non-contact 3D measurement machine used for the measurements is based on the Focus-Variation principle. The technique of Focus-Variation combines the small depth of focus of an optical system with vertical scanning to provide topographical from the variation of focus. Since the system has a limited depth of field only small parts of the object are imaged sharply at the same time. To perform a complete detection of the surface with full depth of field, the precision optics is moved vertically along the optical axis 
while continuously capturing data from the surface. This means that each region of the object is sharply focused. Algorithms convert the acquired sensor data into 3D information with full depth of field. This is achieved by analysing the variation of focus along the vertical axis [Nayar et al. 1994] [Danzl et al. 2011].

A detailed scheme of the measurement principle is depicted in Fig. 1.

The vertical resolution of the system depends on the chosen objective and can be as low as $10 \mathrm{~nm}$. The vertical scan range depends on the working distance of the objective. In contrast to other techniques, the vertical resolution is achieved regardless the scan height leading to a vertical dynamic of 1: 430000. The $X Y$ range is determined by the used objective and typically ranges from $0.14 \times 0.14 \mathrm{~mm}$ to $5 \times 5 \mathrm{~mm}$ for a single field of view. The system is used with a ring light illumination which allows the measurement of slope angles exceeding $87^{\circ}$. In addition to the 3D data, the measurement device also delivers true colour information for each measurement position, which is registered to the height data. This is possible because the system is also a reflection optical microscope.

Basically, Focus-Variation is applicable to surfaces with a large range of different optical reflectance values. As the optical technique is very flexible in terms of using light, typical limitations such as measuring surfaces with strongly varying reflection properties even within the same field of view can be avoided. Specimen can vary from shiny to diffuse reflecting, from homogeneous to compound material and from smooth to rough surface properties. The system overcomes the aspect of limited measurement capabilities in terms of reflectance by a combination of modulated illumination, controlling the sensor parameters and integrated polarization. Focus-Variation is covered by the EN ISO 25178 standard for surface texture measurement [ISO/CD 25178-606: 2015].

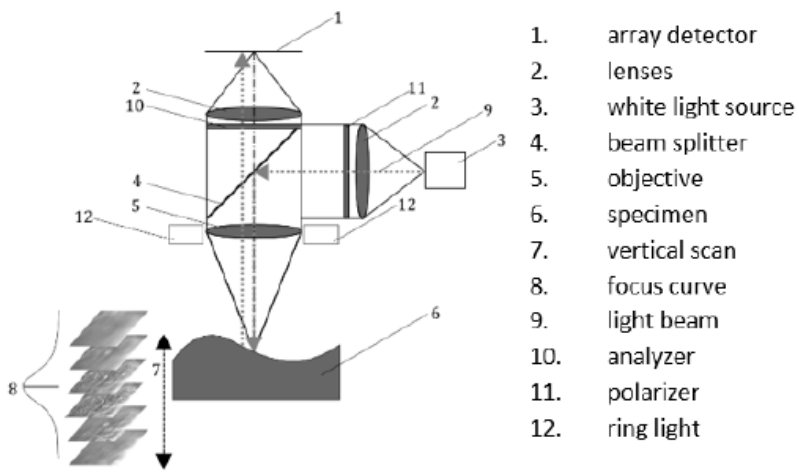

Fig. 1: Scheme diagram of a measurement device based on Focus-Variation [Schuth et al. 2017]

\subsection{Hardware setup}

The basis of the hardware setup is a robust robot cell which contains of a high-resolution Focus-Variation optical 3D sensor, a collaborative six-axis robot with a collaborative gripper and a sample storage for a highly automated workflow. The ring light illumination Sensor, IF-SensorR25 is mounted on a fixed column and a collaborative six-axis robot which is integrated into a fully automated measuring process is picking the samples out of the storage and placing them under the measurement device (see Fig. 2). When the robot reaches his correct position, a signal is triggered that starts the automatic 3D data acquisition and the analysis afterwards. By implementing the communication of the flexible robot and the $3 \mathrm{D}$ measuring device an autonomous operation and control of the measurements is achieved. Focus-Variation is used because it is much more tolerant against vibrations like other optical metrology which is important regarding the vibrations of the UR Robots [Lankmair et al. 2018] [Leach 2020] [Pollak et al. 2020] [Weckenmann 2012] [ISO 15066: 2016]. The analysis of the measured surface texture parameters is giving feedback about the quality of the machining process.

For the further comparison a InfiniteFocusSL was used, which has a similar sensor head but is equipped with a high precision, vibration absorbing $x y$ stage using linear encoders.

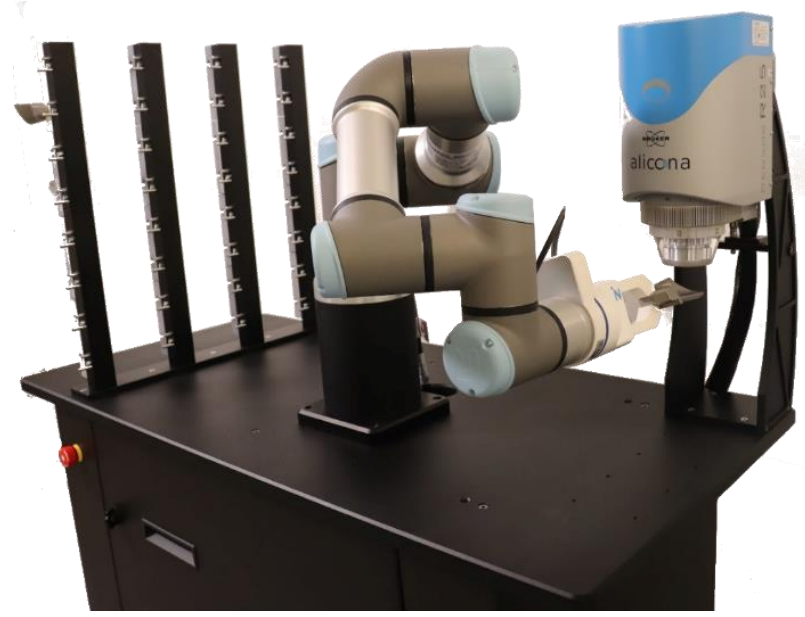

Fig. 2: The hardware setup of the Pick-and-place system

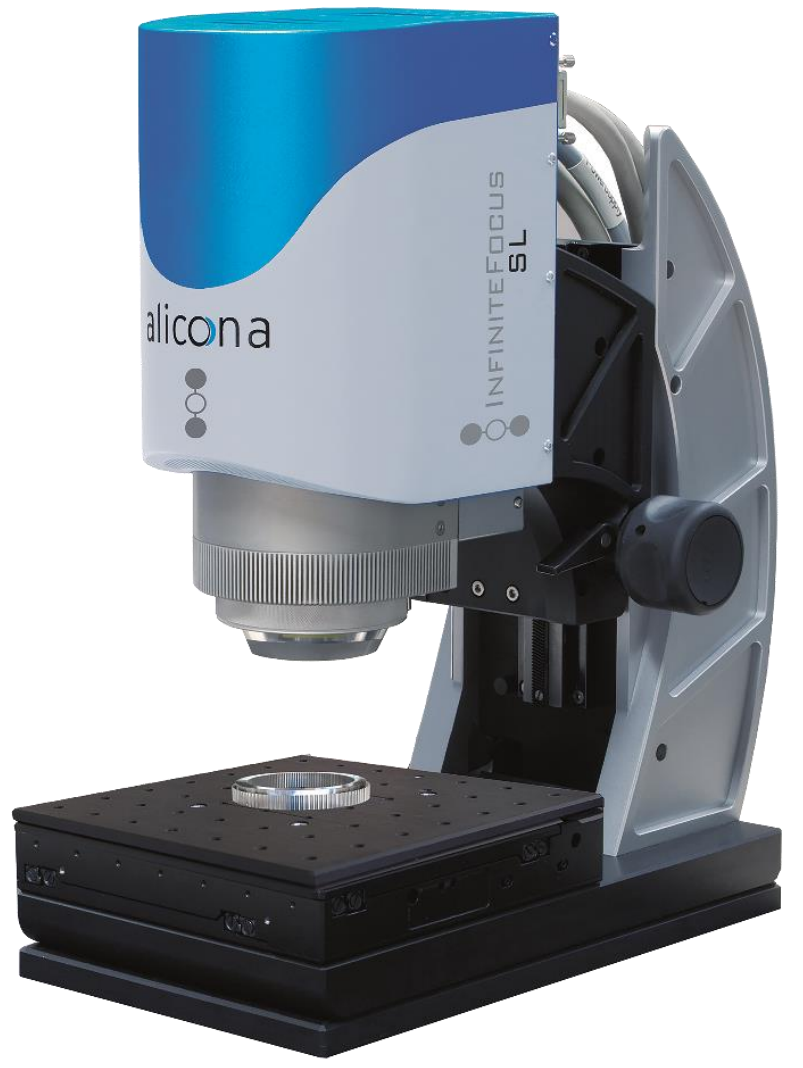

Fig. 3: The hardware setup of the InfiniteFocusSL

The used objective was a $10 x$ lens which has a numerical aperture of 0,3 , a working distance of $17,5 \mathrm{~mm}$, a lateral measurement area of $2 \times 2 \mathrm{~mm}$ and a used vertical resolution of

$100 \mathrm{~nm}$. 


\begin{tabular}{|l|l|}
\hline Vertical resolution & $100 \mathrm{~nm}$ \\
\hline Lateral resolution & $3,91 \mu \mathrm{m}$ \\
\hline Measurement area & $1,4 \mathrm{~mm} \times 1,4 \mathrm{~mm}$ \\
\hline Number of 3D points & $\sim 2000000$ \\
\hline Lambda C Value & $800 \mu \mathrm{m}$ \\
\hline
\end{tabular}

Tab. 1: Measurement settings

\subsection{The used certified standard and sample}

The used tool for the tests was an artefact, the ArealRoughnessTool (see Fig. 4), which was calibrated by the NPL. This artefact has a 1,5 $\mathrm{mm} \times 1,5 \mathrm{~mm}$ nominally isotropic area for the verification of optical measurement systems using surface texture (see Tab. 1) and evaluate the performance of the 3D data acquisitions compared to the certified values [GUM 1993].

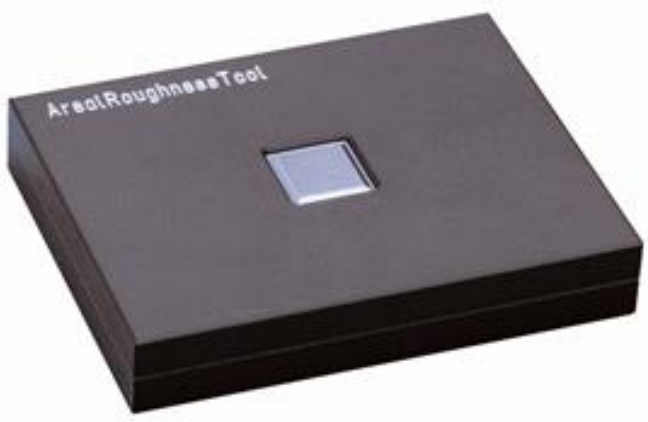

Fig. 4: The certified standard used (calibrated by the NPL); The measurement area is in the middle of the tool

The certified values are the $\mathrm{Sa}$, the arithmetical average deviation of all surface height values

$S a=\frac{1}{A} \int_{A}|z(x, y)| d x d y$

And the Sq, the root-mean-square (RMS) deviation of all surface height values [ISO 25178-2: 2012].

$S q=\sqrt{\frac{1}{A} \iint_{A} z^{2}(x, y) d x d y}$

\begin{tabular}{|l|l|}
\hline Description & Values \\
\hline Calibrated Sa & $753 \mathrm{~nm}$ \\
\hline Uncertainty for Sa & $\mathrm{U}=50 \mathrm{~nm} \mathrm{k}=2$ \\
\hline Calibrated Sq & $947 \mathrm{~nm}$ \\
\hline Uncertainty for Sq & $\mathrm{U}=50 \mathrm{~nm} \mathrm{k}=2$ \\
\hline $\begin{array}{l}\text { Coefficient of thermal } \\
\text { expansion }\end{array}$ & $(4,5 \pm 0,5) 10^{-6} \mathrm{~K}^{-1}$ \\
\hline Calibration laboratory & $\begin{array}{l}\mathrm{NPL} \text { (National Physical } \\
\text { Laboratory) }\end{array}$ \\
\hline
\end{tabular}

Tab. 1: Technical specifications of the certified artefact

In addition to the calibrated artefact a real sample, a turbine blade (see Fig. 5), was used to analyse the capabilities of the measurement setup. The measurement position on this titanium freeform surface was at the tip of the leading edge.

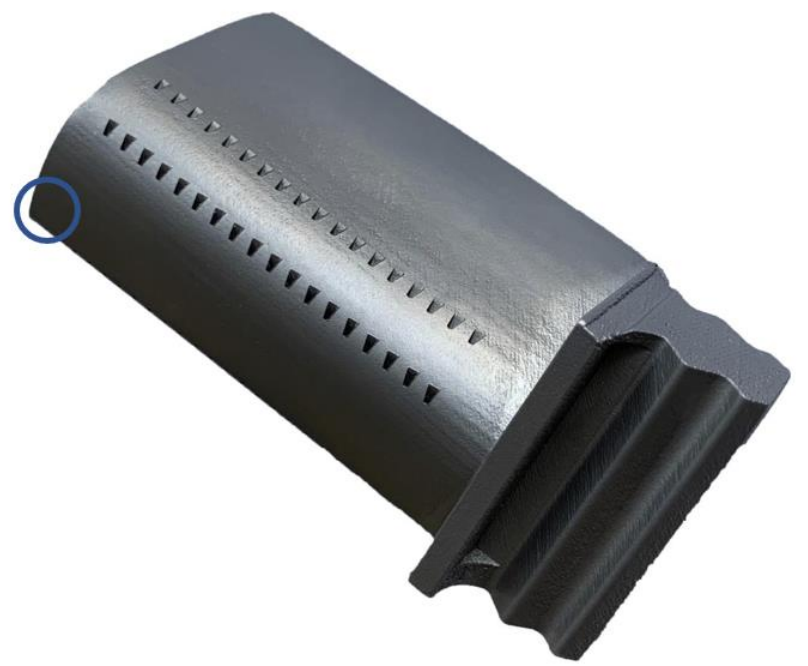

Fig. 5: The sample and the measurement position, circled in blue

\subsection{Measurement workflow}

The measurement workflow was for both systems similar. It was a MSA type-1 study with 25 repetitions for each system and each sample [Dietrich et al. 2014].

For the Pick-and-place system the automatic workflow was:

1. Picking the sample out of the storage

2. Placing it under the sensor head

3. Performing the 3D measurement

4. Putting the sample back into the storage and opening the gripper

5. Repeat step 1-4 till the 25 measurements are finished

For the InfiniteFocusSL the automatic workflow was:

1. Placing the sample on the $x y$-stage

2. The $x y$-stage moves to the measurement position

3. Performing the 3D measurement

4. Moving the $x y$-stage and $z$ axis to a sample change position

5. Repeat step 1-4 till the 25 measurements are finished

\section{COMPARISON OF THE MEASUREMENT SETUPS}

For the comparison of the two different measurement setups an approach was chosen to clarify the capabilities of the Pick-and-place system in comparison with a standard Focus-variation sensor with a high precision motorized $x y-$ stage. The aim of this was to analyse if the gripper, the unprecise robot positioning of $\pm 0,05 \mathrm{~mm}$ and the vibrations in the measurement setup is still sufficient to perform repeatable high resolution surface texture measurements in an automatic way [Gaspar et al 2018] [Pollak et al. 2020] [Sciavicco et al. 2012]. For both Sensor heads the same objective with a 10 times magnification was used.

For the comparison measurements on both features were performed. Each system made 25 complete measurements on each feature and after that the 3D datasets were analysed. For the evaluation of the datasets the SurfaceTextureMeasurement of the Alicona MeasureSuite was used. For filtering the roughness dataset, the standard linear planar Gaussian filter Order 1 with the cut-off value Lc 0,8 mm was chosen [ISO 16610-61: 2010] [ISO 251782: 2012]. The results of these analyses were compared with the values which were calibrated by the NPL (see Tab. 1) and the turbine blade sample [ISO 25178-2: 2012]. 

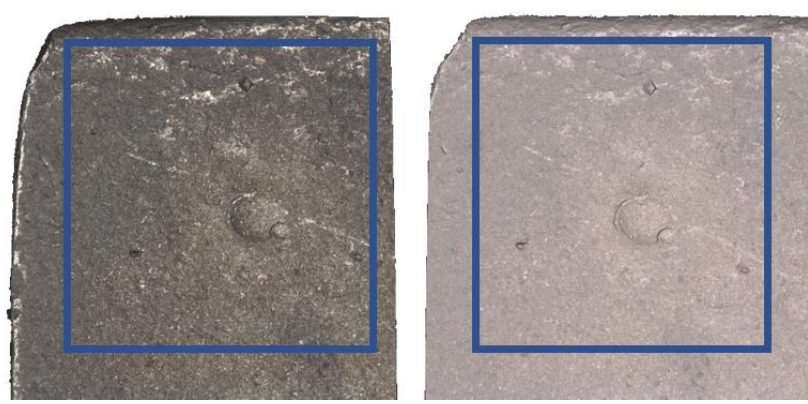

Fig. 6: A dataset of the Pick-and-place setup (left) and of the InfiniteFocusSL (right) aligned for the surface texture measurement, measurement area inside the blue square

\subsection{Evaluation of the certified standard}

The mean Sa values of the surface texture measurements performed at the certified standard are within the certified range. A higher offset of $-20 \mathrm{~nm}$ with the Pick-and-place system in comparison to the $-12 \mathrm{~nm}$ of the InfiniteFocusSL can be observed [ISO 25178-2: 2012].

\begin{tabular}{|l|l|l|}
\hline Description & Unit & Values \\
\hline Calibrated Sa & $\mathrm{Nm}$ & $\begin{array}{l}753 \pm \mathrm{U}= \\
50 \mathrm{~nm}(\mathrm{k}=2)\end{array}$ \\
\hline $\begin{array}{l}\text { Measured Sa } \\
\text { InfiniteFocusSL }\end{array}$ & $\mathrm{Nm}$ & 741 \\
\hline $\begin{array}{l}\text { Measured Sa } \\
\text { Pick-and-place }\end{array}$ & $\mathrm{Nm}$ & 733 \\
\hline
\end{tabular}

Tab. 2: Certified standard, Results of the Sa measurement of the InfiniteFocusSL and the Pick-and-place system

Each Sa value of the 25 3D datasets of each measurement setups was inside the range of the certified standard.

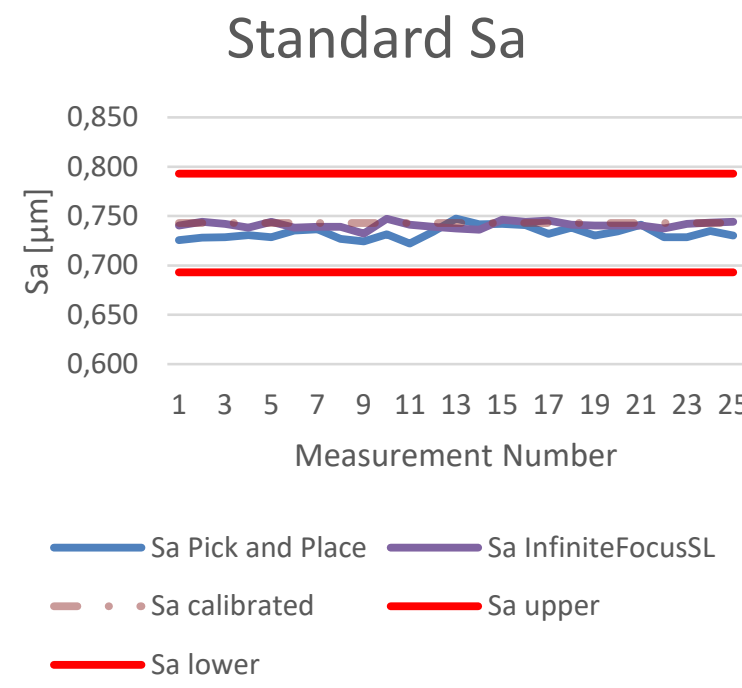

Tab. 3: Certified standard, Results of the Sa measurement of the Pick-and-place and the InfiniteFocusSL

Similar behaviour can be observed regarding the $\mathrm{Sq}$ measurements. The deviation of the mean values are with the InfiniteFocusSL $+2 \mathrm{~nm}$ and with the Pick-and-place system $-12 \mathrm{~nm}$. Both values are inside the calibrated range [ISO 25178-2: 2012].

\begin{tabular}{|l|l|l|}
\hline Description & Unit & Values \\
\hline Calibrated Sq & $\mathrm{nm}$ & $947 \pm \mathrm{U}=$ \\
& & $50 \mathrm{~nm}(\mathrm{k}=2)$ \\
\hline
\end{tabular}

\begin{tabular}{|l|l|l|}
\hline $\begin{array}{l}\text { Measured Sq } \\
\text { InfiniteFocusSL }\end{array}$ & $\mathrm{nm}$ & 949 \\
\hline $\begin{array}{l}\text { Measured Sq } \\
\text { Pick-and-place }\end{array}$ & $\mathrm{nm}$ & 935 \\
\hline
\end{tabular}

Tab. 4: Certified standard, Results of the Sq measurement of the InfiniteFocusSL and the Pick-and-place system

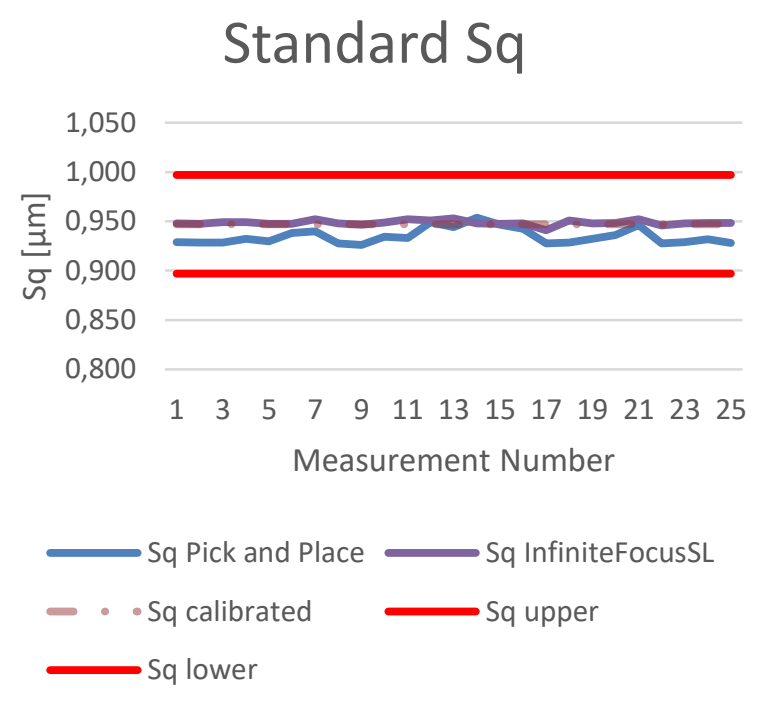

Tab. 5: Certified standard, Results of the Sq measurement of the Pick-and-place and the InfiniteFocusSL

An example of two different datasets in one coordinate system to depict the rough differences is shown at Fig. 7 .

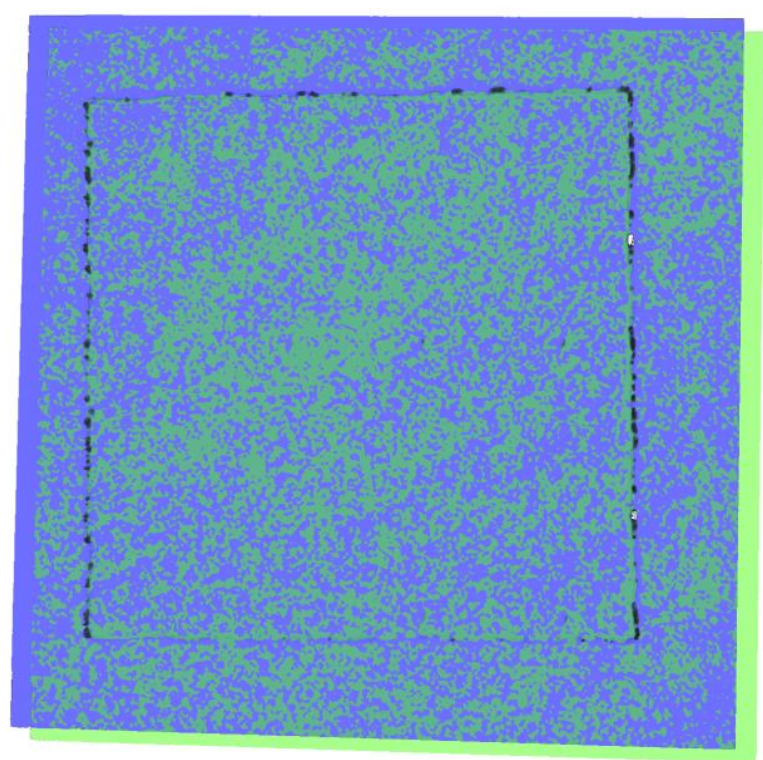

Fig. 7: A Two 3D measurements, one made using the Pickand-place (blue) and one using the InfiniteFocusSL (green) superimposed in one coordinate system

A difference measurement of two datasets, measured by the Pick-and-place System and the InfiniteFocusSL, using the DifferenceMeasurement of the MeasureSuite to compare and visualize the detailed variation of the data acquisition, was performed. The differences are shown in pseudo colour in a height range of -1 to $+1 \mu \mathrm{m}$. The mean deviation of the datapoints used in the difference measurement shows that $96.3 \%$ of the surface are within a $0,5 \mu \mathrm{m}$ tolerance with a mean deviation of $0.01 \mu \mathrm{m}$ (see Fig. 8). 


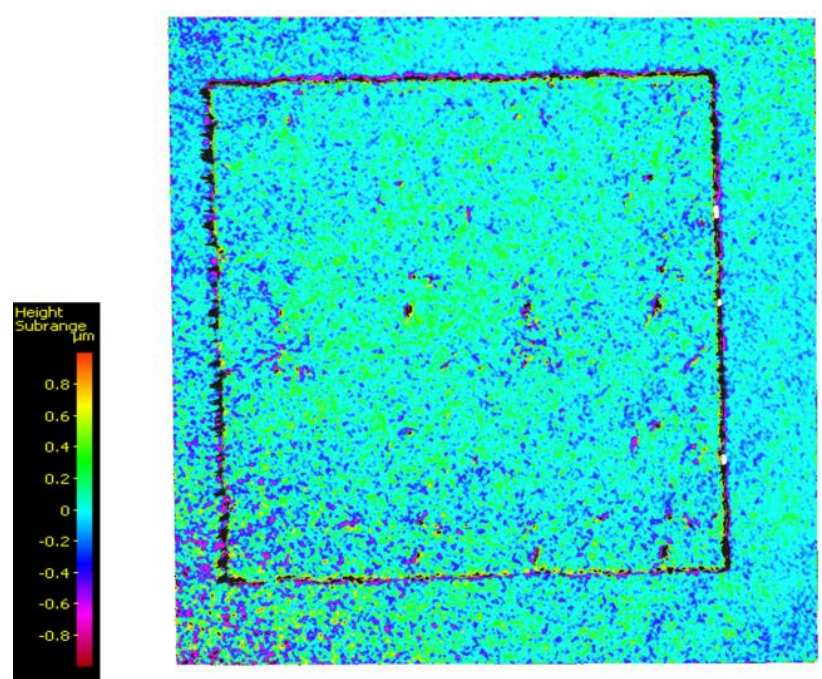

Fig. 8: A difference measurement of the two $3 D$ datasets in pseudo colour (colour range $\pm 1 \mu \mathrm{m}$ ). The rectangle is the surface texture measurement area

\subsection{Evaluation of the sample}

The mean Sa values of the surface texture measurements performed at the turbine blade sample are within a very narrow range. The standard deviation with the InfiniteFocusSL measurements was within the 25 repetitions $2 \mathrm{~nm}$ with a range of $4 \mathrm{~nm}$. The Pick-and-place system in comparison to the InfiniteFocusSL had an offse of $-14 \mathrm{~nm}$ with a standard deviation of $4 \mathrm{~nm}$ and a range of $14 \mathrm{~nm}$.

\begin{tabular}{|l|l|l|}
\hline Description & Unit & Values \\
\hline $\begin{array}{l}\text { Measured Sa } \\
\text { InfiniteFocusSL }\end{array}$ & $\mathrm{nm}$ & 1175 \\
\hline $\begin{array}{l}\text { Measured Sa } \\
\text { Pick-and-place }\end{array}$ & $\mathrm{nm}$ & 1161 \\
\hline
\end{tabular}

Tab. 6: Turbine blade sample, Results of the Sa measurement of the InfiniteFocusSL and the Pick-andplace system

Each Sa value of the $253 \mathrm{D}$ datasets of each measurement setups was inside the range of $\pm 50 \mathrm{~nm}$ which was suitable for the certified standard.

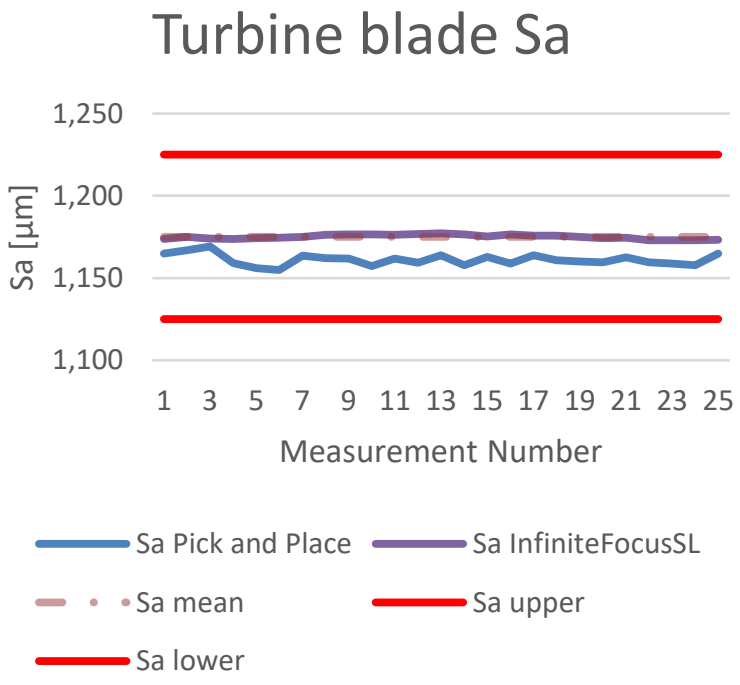

Tab. 7: Sample, Results of the Sa measurement of the Pickand-place and the InfiniteFocusSL
Similar behaviour can be observed regarding the $\mathrm{Sq}$ measurements. The standard deviation with the InfinteFocusSL measurements was within the 25 repetitions $3 \mathrm{~nm}$ with a range of $4 \mathrm{~nm}$. The Pick-and-place system in comparison to the InfiniteFocusSL had an offset of $2 \mathrm{~nm}$ with a standard deviation of $8 \mathrm{~nm}$ and a range of 16 $\mathrm{nm}$.

\begin{tabular}{|l|l|l|}
\hline Description & Unit & Values \\
\hline $\begin{array}{l}\text { Measured Sq } \\
\text { InfiniteFocusSL }\end{array}$ & $\mathrm{nm}$ & 1697 \\
\hline $\begin{array}{l}\text { Measured Sq } \\
\text { Pick-and-place }\end{array}$ & $\mathrm{nm}$ & 1699 \\
\hline
\end{tabular}

Tab. 8: Sample, Results of the Sq measurement of the InfiniteFocusSL and the Pick-and-place system

Also, here each $\mathrm{Sq}$ value of the $253 \mathrm{D}$ datasets of each measurement setups was inside the range of $\pm 50 \mathrm{~nm}$ which was suitable for the certified standard.

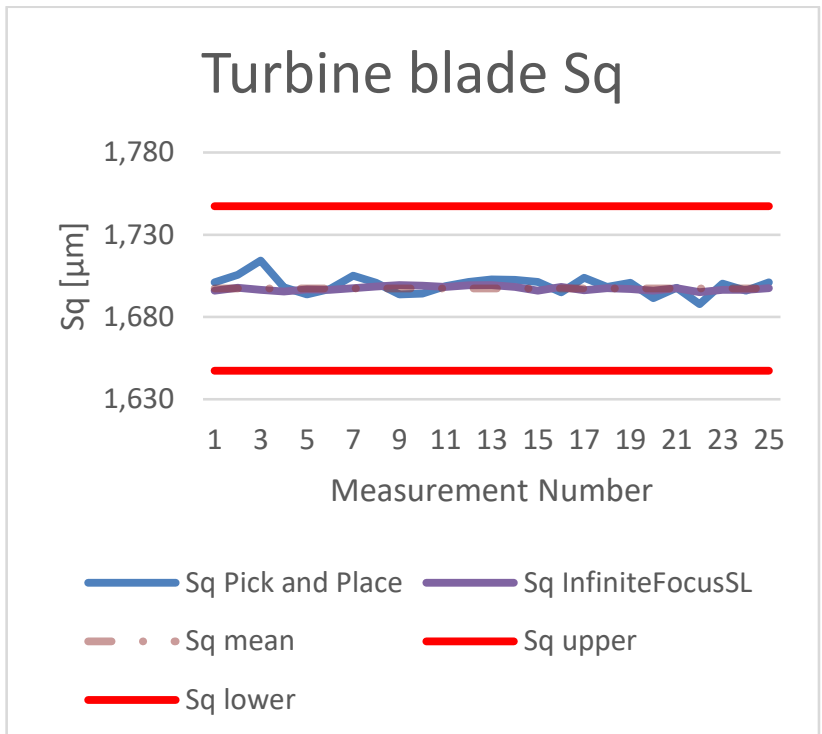

Tab. 9: Sample, Results of the Sq measurement of the Pickand-place and the InfiniteFocusSL

An example of two different datasets in one coordinate system to depict the rough differences is shown in Fig. 9. Here a difference in positioning is more obvious due to the more complex geometry of the turbine blade sample. But the measurement area was similar (see Fig. 6).

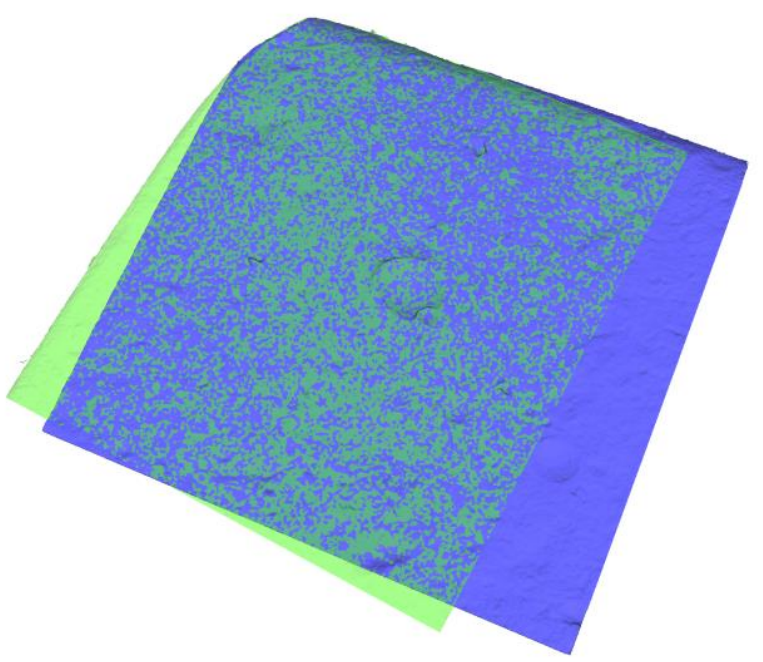

Fig. 9: Two 3D measurements, one made using the Pick- 
and-place (blue) and one using the InfiniteFocusSL (green) superimposed in one coordinate system

A difference measurement of two datasets, measured by the Pick-and-place system and the InfiniteFocusSL, using the DifferenceMeasurement of the MeasureSuite to compare and visualize the detailed variation of the data acquisition, was performed. The differences are shown in pseudo colour in a height range of -1 to $+1 \mu \mathrm{m}$. The mean deviation of the datapoints used in the difference measurement shows that $94.4 \%$ of the surface are within a $0,5 \mu \mathrm{m}$ tolerance with a mean deviation of $0.035 \mu \mathrm{m}$ (see Fig. 10).

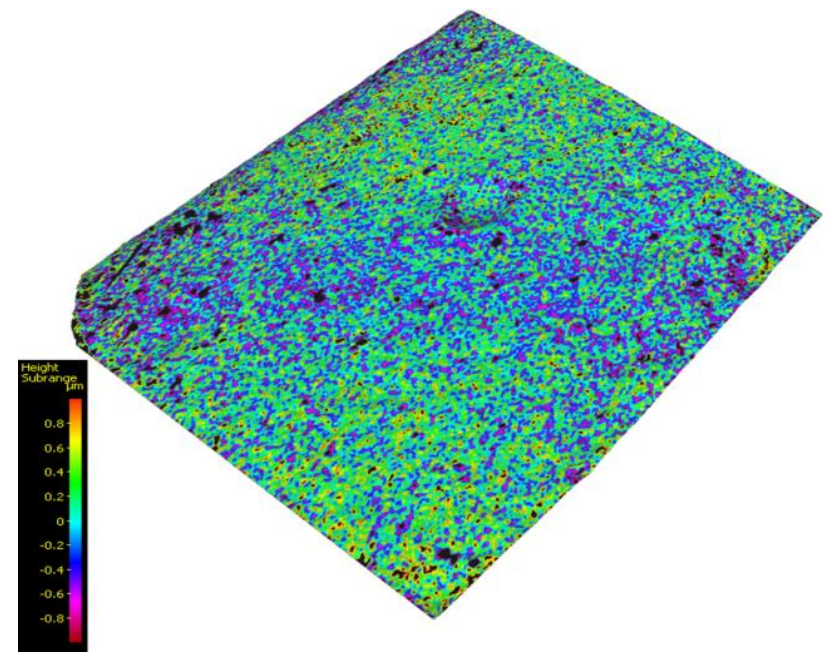

Fig. 10: A difference measurement of the two $3 D$ datasets in pseudo colour (colour range $\pm 1 \mu \mathrm{m}$ )

\section{SUMMARY}

The shown pick-and-place application is capable of performing automatic high resolution optical 3D surface texture measurements using an Alicona Focus-Variation sensor. All acquired 3D datasets and the resulting $\mathrm{Sa}$ and $\mathrm{Sq}$ values were within the specifications of the certified standard calibrated by the NPL. Also, the results of the sample turbine blade demonstrated the possibilities and flexibility of this measurement setup regarding the $\mathrm{Sa}$ and Sq values compared to a standard Focus-Variation system with a motorized $x y$ - table.

All measurements have shown that a certain offset and a higher deviation between the standard system and the robot systems are occurring. These facts are within the certified range of the surface texture standard and might be due to the lower repeatability when using the robot system compared to a high precision $x y$ - stage and because of vibrations caused by the robot.

\section{REFERENCES}

[Danzl et al. 2011] Danzl R., Helmli F., Scherer S., Focusvariation - A robust technology for high resolution optical 3D surface metrology, Journal of Mechanical Engineering, 2011; 57 :245-56
[Dietrich et al. 2014] Dietrich, E., Schulze, A.: Statistische Verfahren zur Maschinen- und Prozessqualifikation. Carl Hanser Verlag GmbH Co KG, 2014, 354-364. ISBN 9783446440555

[GUM 1993] GUM - Guide to the Expression of Uncertainty in Measurement. International Standardization Organization, Geneva, 1993

[ISO 9283: 1998] ISO 9283, Manipulating industrial robots - Performance criteria and related test methods, 1998

[ISO 15066: 2016] ISO 15066, Roboter und

Robotikgeraete - Kollaborierende Roboter, 2016

[ISO 16610-61: 2010] ISO 16610-61, Geometrical Product Specification (GPS) - Filtration Part 61: Linear areal filters: Gaussian filters, 2010

[ISO 25178-2: 2012] ISO 25178-2 Geometrical product specifications (GPS) - Surface texture: Areal - Part 2: Terms, Definitions and Surface texture Parameters, 2012

[ISO/CD 25178-606: 2015] ISO/CD 25178-606, Geometrical product specification (GPS) - Surface texture: Areal Part 606: Nominal characteristics of non-contact (Focus-Variation) instruments, 2015

[Lankmair et al. 2018] Lankmair, T., Riedl M., Schreink M., Danzl R., 2. VDI-Fachtagung Multisensorik in der Fertigungsmesstechnik 2018, Frankenthal, 2018, 235-241. ISBN 9783180923260

[Leach 2011] Leach R.K., Optical Measurement of Surface Topography. Berlin, Springer-Verlag, 2011. ISBN 9783642120114

[Leach 2014] Leach R.K., Fundamental Principles of Engineering Nanometrology. 2nd ed., Elsevier, 2014. ISBN 9780128101537

[Leach 2020] Leach R.K., Advances in Optical Surface Texture Metrology. IOP Publishing, 2020, 49-78. ISBN 9780750325264

[Nayar et al. 1994] Nayar, S. K., Nakagawa, Y., Shape from focus. IEEE Transactions on Pattern Analysis and Machine Intelligence, 1994, Vol. 16, No. 8, 824-831

[Pollak et al. 2020] Pollak M., Kocisko M., Paulisin D., Baron $P$., Measurement of unidirectional pose accuracy and repeatability of the collaborative robot UR5. Advances in Mechanical Engineering, 2020, Vol. 12 1-21

[Schuth et al. 2017] Schuth M., Buerakov W., Handbuch Optische Messtechnik: Praktische Anwendungen für Entwicklung, Versuch, Fertigung und Qualitätssicherung. Carl Hanser Verlag GmbH Co KG, 201. ISBN 9783446436343

[Sciavicco et al. 2012] Sciavicco L., Siciliano B., Modelling and Control of Robot Manipulators. Berlin, Springer-Verlag, 2012

[Weckenmann 2012] Weckenmann, A., Koordinatenmesstechnik - Flexible Strategien für funktions- und fertigungsgerechtes Prüfen. 2nd ed. Hanser Verlag, 2012. ISBN 9783446407398

[Zaborowski et al. 2019] Zaborowski T, Panda A, Androvic L., Robots and cobots, main differences. Studia Mater 2019; 39: 4-8. 